\title{
Effectiveness of Educational Nursing Strategies on Nurse's Knowledge and Skills regarding Traumatic Head Injury in Children
}

\begin{abstract}
Traumatic head injury is a pressing public health problem leading to disability and death in children and adolescents worldwide. Aim: This study aimed to assess the effectiveness of educational nursing strategies on nurse's knowledge and skills regarding traumatic head injury in children. Setting: The study was conducted in the Paediatric Intensive Care Unit and Emergency Department at Birket El Sabah Central Hospital, Menoufia Governorate, Egypt. Methods: A quasi-experimental design was carried out for this study (pre/post \& follow-up). A convenience sample of 40 nurses provided direct nursing care to the children with traumatic head injury in the above-mentioned setting was selected from February to October 2019. Two tools were used for data collection, tool one: A structured interviewing questionnaires, it consisted of 7 sections to assess nurses' knowledge. Tool two: An observational checklist to assess the nurses' practice of the Glasgow coma scale. Results: The results of this study revealed that there was a high statistical significance difference between pre, post and follow up tests regarding nurses' knowledge and skills of children with traumatic brain injury at $1 \%$ level of statistical significance. Conclusion, Implementation of educational nursing strategies improved nurses' knowledge and skills while managing children with traumatic head injury. Recommendation, Continuous educational programs should be scheduled on a constant base for nurses' about traumatic brain injury to enhance children's quality of care and improve their outcome.
\end{abstract}

Keywords: Educational nursing strategies, Nurses, Knowledge, Skills, Children, Traumatic head injury DOI: $10.7176 / \mathrm{JHMN} / 69-12$

Publication date: December $31^{\text {st }} 2019$

\section{Introduction}

Traumatic head injury (TBI) is a silent epidemic disease leading to death and disability in children and adolescents worldwide (Ismail, 2019 \& Hussein, 2018). It's a structural injury and physiologic disruption of brain function caused by blunt trauma, acceleration, deceleration forces or exposure to blast, which leads to death (Amaranath et al., 2014 \& Widerstrom et al., 2009). World Health Organization (WHO) referred to TBI as a public health crisis due to its growing incidence, social, economic burden to hospitalizations and utilization of intensive care units (Alsolamy et al., 2017 \& Gennai et al., 2015). Globally, in every 15 second head injury occurs and in every 12 minutes patient dies from TBI (Yattoo et al., 2009). Annually, it is estimated that over 10 million people sustain a traumatic brain injury around the world (Oyesanya et al., 2018).

World Health Organization expected that by 2020, traffic accidents will be the third greatest cause of disease and injury that occur from motor-vehicle use in low-income and middle-income countries (Shehab et al, 2018 \& Gennai et al., 2015). TBI accounts for approximately 50\% of all deaths in children and almost 1 in every 6 requires treatment in the emergency department (Maconochie, 2003). The incidence in children between 0 and 18 years is 280-1373 per 100,000, most of them are young children between 0 and 4 years, followed by adolescents between 15 and 19 years (Koepsell et al., 2011). Males are 1.5 times as expected as females to sustain traumatic brain injury (Seliman, et al., 2014 \& Faul et al., 2010). In United States, motor vehicle collisions and falls are the most frequent precipitating events resulting in over 500,000 emergency department visits, 37,000 hospitalizations and over 2000 deaths annually in children younger than 14 years old (Borich et al., 2013 \& Langlois et al., 2004). In India, approximately 1.5-1.7 million patients are neurologically disabled from TBI reported each year (Das et al., 2012).

In Egypt, injuries are a significant source of mortality and morbidity. It is considered the fifth leading cause of death. Each year about 12,000 Egyptians died from road traffic accidents with a rate of 60 victims per day based on the latest statistics carried out by the Egyptian Central Agency for Mobilization and Statistic Egyptian Central Agency, 2016 \& Hassanein et al., 2018. TBI is non-degenerative and non-congenital abnormalities result in a combination of physical, cognitive and behavioral impairment due to externally inflict trauma (Kutzleb, 2012 \& Satyanegara, 2010). The severity is determined by indicators such as Glasgow Coma Scale Score, loss consciousness, length and presence of post-traumatic amnesia (Teasdale et al., 2014). There are different types of 
TBI (mild, moderate, and severe) that cause different types of impairments (Teasdale et al., 2014). Also, it can be either closed or open (penetrating) (Amaranath et al., 2014). TBI is divided into two phases, primary and secondary. Primary brain injury is the physical damage to parenchyma (tissue, vessels) that occurs during a traumatic event, resulting in shearing and compression of the surrounding brain tissue (Unterberg et al., 2004). It progresses over hours, days and weeks after direct head injury. Disabilities occur due to neuronal destruction and cerebral ischemia from brain swelling, hematoma formation, hypoxia and hypotension (Finnie and Blumbergs, 2002, Murthyet al., 2005 \& Granacher, 2015).

Secondary brain injury resulting from the constellation of cellular and biochemical processes over the subsequent hours and days including cerebral edema, hematomas, hydrocephalus, vasospasm, metabolic derangement, infection and seizure (Ismail et al., 2019., Guarg et al., 2017, Haddad \& Arabi, 2012). Accurate assessment of neurological status in children and early initiation of critical care is vital to prevent deaths (Paul, 2012). The Glasgow Coma Scale (GCS) is one of the most effective and prevalent scoring technique to describe the level of consciousness (Falk et al., 2014). The ultimate goal in the use of GCS is to recognize those in need of acute intervention as early as possible and prevent secondary brain injuries, thereby reducing both mortality and morbidity among children (National institute for health and clinical excellence, 2013). Glasgow Coma Scale based on the sum of several categories ranging between 3 and 15 according to responsiveness of general verbal, visual and motor stimuli calculated from observed spontaneous activities and use of verbal and/or painful stimulus (Hopkin et al., 2016 \& Prado, 2015). GCS score of 13-15 indicates a mild brain injury, the majority of patients resolve within 10 to 14 days (McCrory et al., 2017 \& Benson et al., 2011). A score of 9-12 indicates a moderate brain injury and any patient scoring 8 or below indicates severe brain injury (McNarry and Goldhill, 2004). However, post concussive syndrome is a persistence of somatic manifestations such as headache, neck pain, dizziness, nausea, balance dysfunction (Mucha et al., 2014).

Children with traumatic brain injury usually present with a combination of medical, physical, sensory, cognitive, behavioral, communication and social problems. Troubled cognition is the hallmark symptom of traumatic head injury that may lead to difficulties with memory, attention, learning and co-ordination (Godwin et al., 2011). Cognitive manifestations such as memory loss and slow reaction time in addition to psychological disorders such as depression, and anxiety (Ponsford et al., 2019, Dwyer \& Katz, 2018 and Kontos, 2004). Symptoms like headache and dizziness are the most commonly reported, followed by nausea and neck pain (Morin et al., 2016). Initial management in the emergency room starts with recognizing TBI, assess patients' level of consciousness and secure the airway with an endotracheal tube. Children with Glasgow Coma Scale (GCS) score of $\leq 8$, ensure adequate oxygenation $(\mathrm{PaO} 2>60 \mathrm{mmHg}$ ) and $\mathrm{BP}$ (systolic $\mathrm{BP}>90 \mathrm{mmHg}$ ), insert the peripheral intravenous cannula and cardiac monitoring in addition to pulse oximetry (Hemphill \& Phan, 2016 and Sheriff \& Hinson, 2015).

The primary goal of nursing management is to maintain adequate cerebral tissue perfusion (Seliman, 2015 and Jaddoua et al., 2013). Nursing management focuses on promoting ICP stability, minimizing ICP rises, ensuring adequate CPP, avoiding hypotension plus hypoxia and rehabilitation treatment to improve patient outcome in addition to better survival rates (Van et al., 2011., Neumann et al., 2010 and Tume, 2007). Most interventions are planned to reduce symptoms such as headaches, cognitive problems or other post-concussion syndrome (Seliman, 2015 and Jaddoua et al., 2013). Pediatric nurses have a vital role in coordinating care across the recovery continuum in all phases of TBI recovery (Oyesanya, 2018).

The consequences of traumatic brain injury include a significant change in the patient's life course, overwhelming disruption of family dynamics, massive loss of income or earning potential and costly lifetime expenses (Zemek et al., 2013 and Maas et al., 2013). Nurses should provide information and education to reassure patients and family by including a follow-up consultation, Family problem-solving interventions in order to improve child and family functioning (Heugten et al., 2017). Delayed recognition of cognitive, emotional and behavioral problems may lead to unnecessary chronic and disruptive problems in activities and participation (Konigs et al., 2015 and Levin et al., 2008).

\section{Operational definitions}

- Educational nursing strategies: It is a planned educational programme implemented to nurses' in order to improve their knowledge and skills while managing children with traumatic brain injury.

- Nurses: are licensed health care professionals who practices independently or under supervision to promote and maintain traumatic child health.

- Knowledge: It refers to acquaintance with or understanding of a science, art about traumatic head injury.

- Skills: the ability to use knowledge effectively and readily in performance. 
- Children: a young person less than 18 years old.

- Traumatic head injury: is injury of the scalp, skull, or brain range from a minor bump on the skull to serious brain injury.

\section{Aim of the study:}

The aim of this study is to assess the effectiveness of educational nursing strategies on nurse's knowledge and skills regarding traumatic head injury in children.

Research hypothesis: For achieving the aim of this study, the following hypotheses were formulated:

Hypotheses 1: Nurses' knowledge will improve after implementation of educational nursing strategies regarding traumatic head injury in children.

Hypotheses 2: Nurse's skills will improve Post Implementation of educational nursing strategies regarding the management of traumatic head injury.

Hypotheses 3: There will be a positive association between nurses' Knowledge and skills Post Implementation of educational nursing strategies.

\section{Material and Methods \\ Materials:- \\ Research Design:}

The research method adopted for the present study was a quasi-experimental design (pre- post and follow up test) to achieve the aim of the current study.

\section{Setting:}

This study was conducted in the Pediatric Intensive Care Units and Emergency Department at Birket El Sabah Central Hospital, Menoufia Governorate, Egypt.

\section{Sampling}

In this study a convenience sample was used to include all nurses providing direct nursing care to children with traumatic head injury in the above mention setting (40 nurses).

Inclusion criteria: The inclusion criteria for sampling were:

1) Nurses who are willing to participate in the study.

2) Nurses who are available during data collection.

\section{Tool of data collection}

\section{Two tools were used for data collection:}

Tool one: A structured interviewing questionnaire. It was adopted from Hussein, (2018) and modified by the researchers after revising the related literature. It was written in a simple Arabic language .It was used to assess nurse's knowledge and comprises 7 parts:

Part one: Socio-demographic characteristics of the studied sample such as nurses' age, educational level, working areas, years of experience and previous training.

Part two: Questions about anatomy and physiology of the head. This part consisted of 7 multiple choice questions (head structure, Parts of the brain controls personality, behavior \& emotions, basic life functions, visual therapy, cognition, understanding, and storing memories in the brain).

Part three: Questions about traumatic head injury. This part consisted of 5 multiple choice questions (Causes of head injury, clinical manifestations, diagnosis, treatment, and complications).

Part four: Questions about intracranial pressure. This part consisted of 8 multiple choice questions (Normal range, volume of intracranial pressure, content of the skull, causes \& risk factors, signs \& symptoms, diagnostic measures, complications, and nursing management).

Part five: Questions about Glasgow coma scale. This part consisted of 9 multiple choice questions (Function of GCS, components, eye-opening, verbal response, motor response, best result, worst result, degree of coma and documentation).

Part six: Questions about principals of nursing management of head trauma. This part consisted of 8 multiple choice questions (Tracheal suctioning, oxygen therapy, Sao 2 monitoring, arterial puncture, cardiac monitoring, CVP measurement, deep vein thrombosis prophylaxis, and neurological management). 
Part seven: Questions about basic care of head injury. This part consisted of 6 multiple choice questions (side effect of increase metabolic rate, Feeding, changing position, nursing care when ear bleed, nursing care to patient eyes and contraindication of restrain).

\section{Scoring system of the knowledge: \\ Total knowledge scores}

The total scores of nurses' knowledge against the 7 basic items, nurses were given one point for each correct answer and zero for incorrect answers. For each part, the scores of the items were summed up. These scores were converted into a percent. A Total score of $75 \%$ and more was considered satisfactory in knowledge while scores below $75 \%$ was considered unsatisfactory.

Tool two: An observational checklist based on a Pediatric Glasgow coma scale (PGCS). The scale was adopted from Elhagga and Eldesouky, (2016) to assess nurse's practice about PGCS. It contained eye, motor and verbal response. Eye opening includes 4 items, verbal response involves 5 items and motor response contains 6 items. Numeric values stared from 1-4 in eye opening, from1-5 in verbal response and from 1-6 in motor response. In each part the highest numeric value indicates best response and lowest values indicates poor response. The sum of these numeric values referred to child level of consciousness.

\section{Scoring system of the practice: \\ Total practice Scoring system}

The total scores of nurses' practice against three basic items, nurses were given one point for each competent practice and zero for Incompetent practice. For each part, the scores of the items were summed up. These scores were converted into a percent. Incompetent practice: $<85 \%$ of total practice score and competent practice: $\geq 85 \%$ of total practice score.

Approval: A certified consent obtained from the administrators to get permission for data collection after clarifying the aim of the study and intervention which could contribute to improve nurse's knowledge and practice about traumatic brain injury of children.

Ethical considerations: At the initial interview, the researcher introduced themselves to the nurses and clarified to them the aim of the study. Each nurse was informed about the right to refuse or participate in the study with anonymity and confidentiality of the information gathered was ensured. Finally, nurse's formal consent for participations has been obtained and reassured that they had the right to withdraw from the study with no penalty.

\section{Tool development:}

It was adopted from Hussein, (2018) \& El-Naggar, (2014) and modified by the researchers after reviewing related literature regarding paediatric traumatic brain injury, through using, articles, periodicals, books and magazines to be acquainted with the various aspects of the research problem.

Validity: Before starting the data collection, the tool was translated into Arabic and tested for their content validity by a group of three professor experts in pediatric nursing.

Pilot study: A pilot study was carried out on 4 nurses'(10\% of the studied sample) in order to test the clarity and applicability of the tools as well as to evaluate the average time needed to fill the questionnaire. No changes were made to the study tools, so the $10 \%$ of the study sample was included in this study.

Reliability of the tool: - Reliability was assessed by correlation coefficient (0.8).

\section{Procedure for data collection}

Fieldwork: Data collection was conducted from February to October 2019.

\section{Assessment phase}

The researchers introduced their selves to the nurses and clarified the purpose of the study. Each nurse interviewed individually according to the available time. At the beginning of the study, nurses asked to fill out the interview questionnaire (tool1) and it took 15 minutes (pretest). The nurse's Observational checklist was filled out by the researchers to assess nurses' performance while assessing level of consciousness through tool2 (pretest). Nurses were not informed that they were observed. Nurses' performance was observed three days per week during the morning and afternoon shifts. The assessment phase took 3 months to complete the required data. Areas of weaknesses in nurses' knowledge and practices were identified and program objectives were set.

\section{Implementation phase}

Educational nursing strategies (teaching booklet in Arabic language) were designed for nurses who caring for children with traumatic brain injury. It was developed by the researchers after revising current literatures built 
on the assessment of nurses' knowledge obtained from structured interviewing questionnaire (tool 1) and observations obtained from nurses observational checklist while assessing child level of consciousness through using GCS (tool 2). Nurses were divided into groups (each group interviewed two times per week between morning and afternoon shift according to their availability). Every interview lasted for 25 to 30 minutes. Each group contained 3 to 4 nurses. Each group took 2 educational sessions about theoretical \& practical parts as follows: first session includes theoretical knowledge about anatomy and physiology of the head, traumatic brain injury, intracranial pressure, GCS, principals of nursing management and basic care. Practical session includes Glasgow coma scale (eye-opening response, verbal response and motor response). During these sessions, the researchers teach content in a clear, simple language using lectures, illustrative pictures, displayed simple training videos for practical skills related to GCS. At the end of each session researchers close the session with a summary of the main points and giving feedback with positive verbal words. Each nurse received the Arabic teaching booklet including theoretical and practical parts at the end of the last session.

\section{Evaluation phase}

Nurses' knowledge and practices about traumatic brain injury were reassessed immediately after implementation of the educational program and it took 3 months (posttest). Reassessment for retention was carried out 3 months later (follow-up test).

Data processing and analysis: Data were analyzed using the IBM Statistical Package of Social Science (SPSS) version 20. Quantitative data were presented by mean (X) and standard deviation (SD). Qualitative data were presented in the form of frequency distribution tables, number and percentage. Statistical significance was considered at $\mathrm{p}$-value $\leq 0.05$ and $\mathrm{P}<.001$.

Results:

Table 1: Distribution of the studied nurses according to their demographic characteristics. $\mathrm{No}=40$ )

\begin{tabular}{|c|c|c|c|}
\hline \multicolumn{2}{|c|}{ Demographic characteristics } & No & $\begin{array}{c}\text { Percentage } \\
\%\end{array}$ \\
\hline \multirow[t]{4}{*}{ Age } & $20-<30$ years & 22 & $55 \%$ \\
\hline & $30-<40$ years & 17 & $42.5 \%$ \\
\hline & $40-<$ years & 1 & $2.5 \%$ \\
\hline & \multicolumn{3}{|c|}{ Mean \pm SD $29.1 \pm 6.36$} \\
\hline \multirow[t]{3}{*}{ Education Level } & Diploma nursing & 7 & $17.5 \%$ \\
\hline & Technical nursing institute & 17 & $42.5 \%$ \\
\hline & Bachelor degree & 16 & $40.0 \%$ \\
\hline \multirow[t]{4}{*}{ Years of experience } & $1-<5$ years & 10 & $25 \%$ \\
\hline & $5-<10$ years & 15 & $37.5 \%$ \\
\hline & $10-<15$ years & 5 & $12.5 \%$ \\
\hline & $15-<20$ years & 10 & $25 \%$ \\
\hline \multirow[t]{2}{*}{ Working area } & Intensive care unit & 21 & $52.5 \%$ \\
\hline & Emergency room & 19 & $47.5 \%$ \\
\hline \multirow{2}{*}{$\begin{array}{l}\text { Previous training about } \\
\text { traumatic head injury }\end{array}$} & Yes & 0 & $0.0 \%$ \\
\hline & No & 40 & $100 \%$ \\
\hline
\end{tabular}

Table 1 describes distribution of the studied nurses according to their demographic characteristic, it was illustrated that mean \pm SD were $29.1 \pm 6.36$ and more than half of the studied nurses $(55 \%)$ aged between $20-<$ 30 years old while, $42.5 \%$ of them were aged $30<40$ years old. Also, more than half of the studied sample $(52.5 \%)$ working in Intensive care units and $47.5 \%$ of them are working on the Emergency room. 


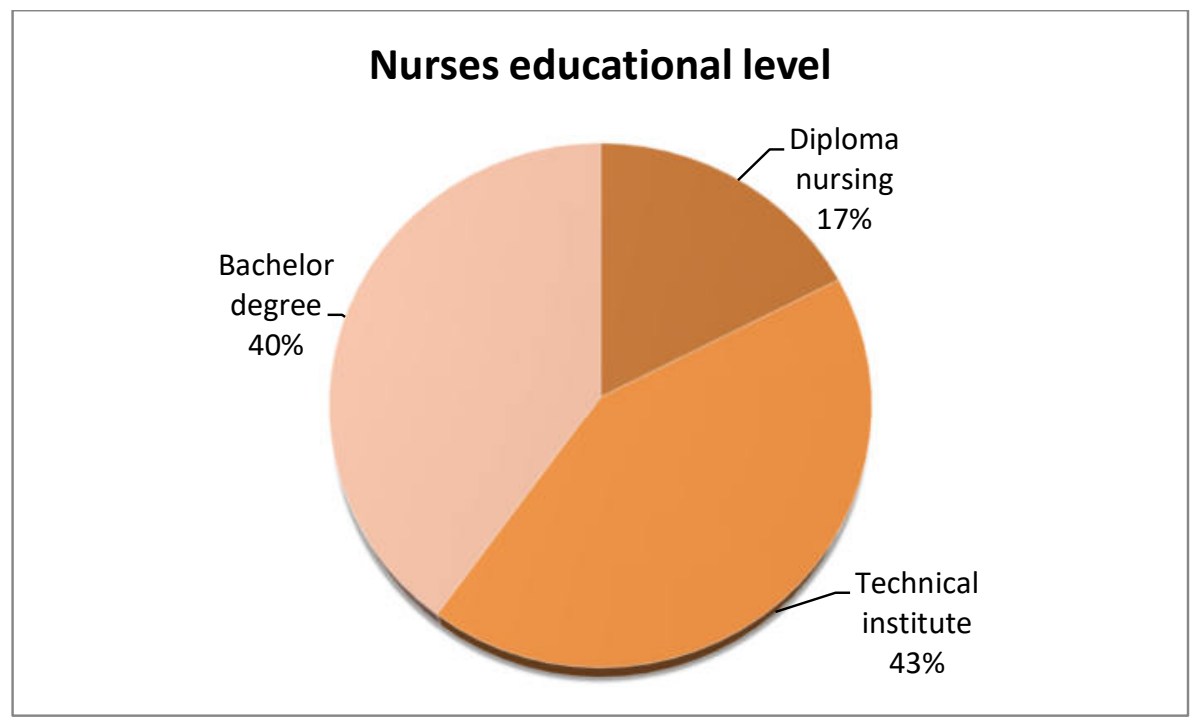

Figure (1): Nurses educational level.

Figure 1 represented nurses educational level, it was clarified that the majority of studied nurses were technical nursing institute and bachelor degree ( $43 \%$ and $40 \%$, respectively) while, the minority of them were diploma nursing( 17\%).

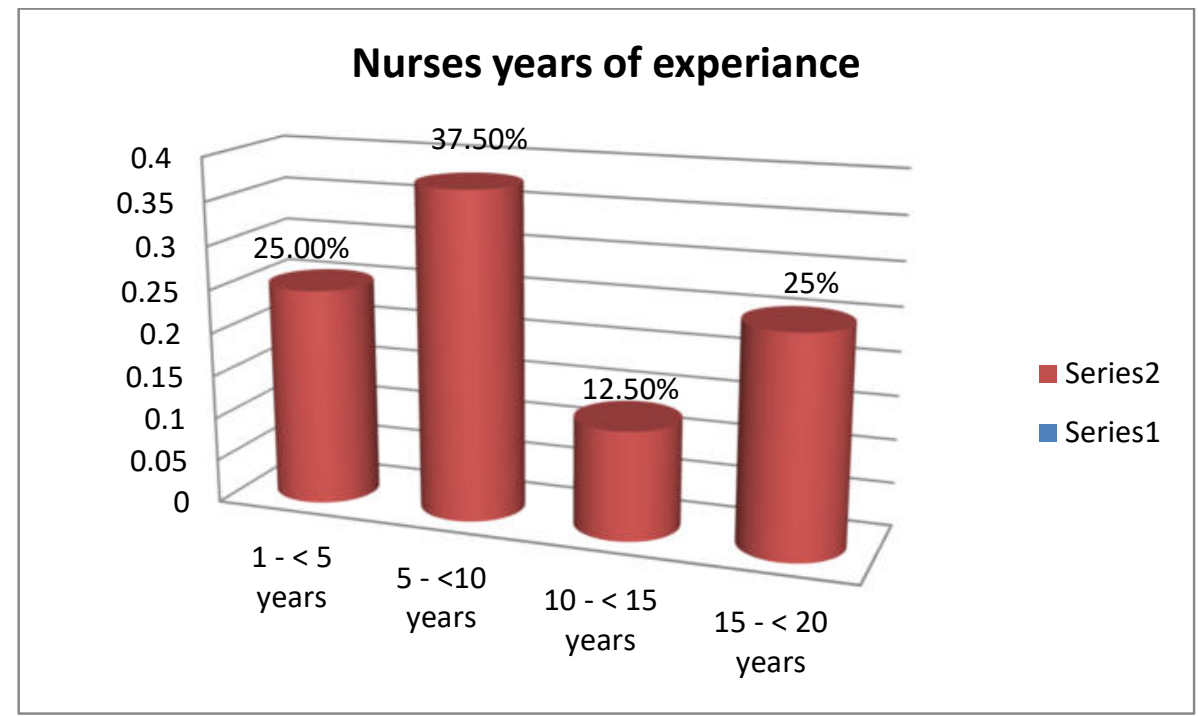

Figure (2):- Nurses 'Years of Experience.

Figure 2 illustrated nurses' years of experience. The figure showed that $37.50 \%$ of studied nurses had $5-<10$ years and $25 \%$ of them had $1-<5$ and $15-<20$ years of experience. 


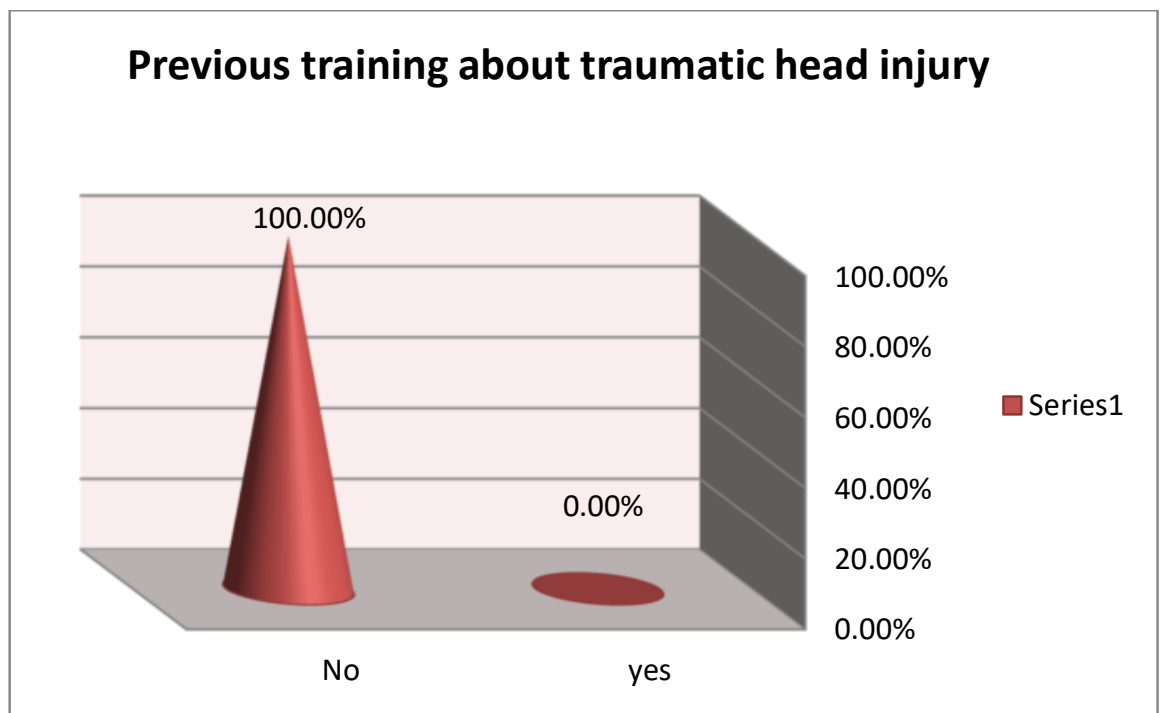

Figure (3):- Previous training about traumatic brain injury

Figure 3 showed previous training about traumatic brain injury. The figure showed that none of the nurses had previous training about traumatic brain injury.

Table (2): Differences in total Mean \pm S.D of nurses' knowledge about traumatic brain injury on pre, post and follow-up tests $(n=40)$.

\begin{tabular}{|c|c|c|c|c|c|}
\hline \multirow[t]{2}{*}{ Knowledge } & Pre & Post & Follow up & \multirow[t]{2}{*}{ F. test } & \multirow{2}{*}{$\begin{array}{c}P . \\
\text { value }\end{array}$} \\
\hline & Mean \pm S.D & Mean \pm S.D & Mean \pm S.D & & \\
\hline $\begin{array}{c}\text { Anatomy and physiology of head } \\
(7 Q)\end{array}$ & $3.75 \pm 2.38$ & $6.50 \pm 0.64$ & $6.2 \pm 0.45$ & 7.05 & $\begin{array}{c}0.001^{*} \\
*\end{array}$ \\
\hline Traumatic brain injury (5Q) & $3.90 \pm 1.30$ & $4.85 \pm 0.53$ & 4the $.3 \pm 0.44$ & 4.28 & $\begin{array}{c}0.001^{*} \\
*\end{array}$ \\
\hline Intracranial pressure (8Q) & $3.03 \pm 0.80$ & $5.60 \pm 0.63$ & $5.1 \pm 0.51$ & 15.97 & $\begin{array}{c}0.001^{*} \\
*\end{array}$ \\
\hline Glasgow coma scale (9Q) & $3.43 \pm 2.49$ & $7.40 \pm 0.71$ & $6.9 \pm 0.46$ & 23.3 & $\begin{array}{c}0.001^{*} \\
*\end{array}$ \\
\hline $\begin{array}{l}\text { Principals of nursing management } \\
(8 \mathrm{Q})\end{array}$ & $5.38 \pm 2.49$ & $8.30 \pm 1.11$ & $7.9 \pm 1.3$ & 6.78 & $\begin{array}{c}0.001^{*} \\
*\end{array}$ \\
\hline Basic care $(5 \mathrm{Q})$ & $5.65 \pm 0.74$ & $7.55 \pm 0.68$ & $7.2 \pm 0.58$ & 12.02 & $\begin{array}{c}0.001^{*} \\
*\end{array}$ \\
\hline
\end{tabular}

$\mathrm{NB}:$ ** $\mathrm{P}<001$

P1: between pre, post-test and follow up test

Table 2: showed differences in total mean \pm S.D of nurses' knowledge about traumatic brain injury on pre, post and follow-up tests, it was clarified that total Mean \pm S.D of knowledge increased on post and follow up test than on pre-test. Therefore, there was a highly statistical significant difference between nurses' knowledge on pre and post-test at $1 \%$ level of statistical significance. 


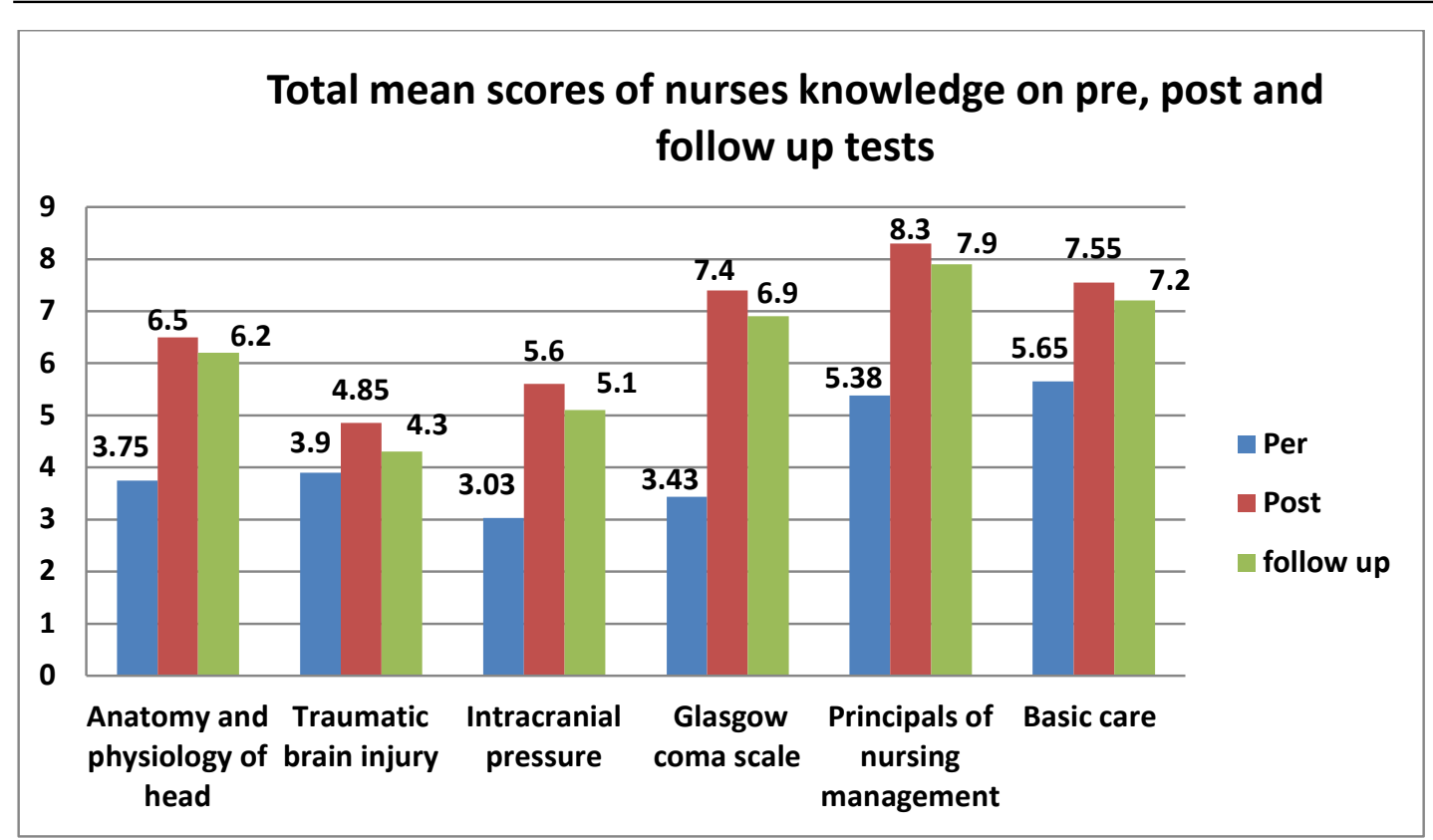

Figure (4): Total mean scores of nurses' knowledge on pre, post and follow-up tests.

Figure 4: showed total mean scores of nurses' knowledge on pre, post and follow-up tests, it was noticed from the results of the present study that the most prominent improvements were in the scores of knowledge about principals of nursing managements, basic care and Glasgow coma scale (8.3, 7.5 and 7.4 respectively). While, there was a reduction in total knowledge scores obtained after three months in all items than on post-test.

Table (3) Comparison between Nurses' Knowledge about principals of nursing management of traumatic brain injury on pre, post and follow-up tests. $(n=40)$

\begin{tabular}{|c|c|c|c|c|c|c|c|c|c|}
\hline \multirow{2}{*}{\multicolumn{2}{|c|}{ Items }} & \multicolumn{2}{|c|}{ Pre } & \multicolumn{2}{|c|}{ Post } & \multicolumn{2}{|c|}{ Follow up } & $X^{2}$ & \multirow{2}{*}{$P$ value } \\
\hline & & & & & & & & & \\
\hline \multirow[t]{2}{*}{ Tracheal suctioning } & Correct & 40 & $100 \%$ & 40 & $100 \%$ & $\begin{array}{l}\text { No } \\
40\end{array}$ & $\begin{array}{c}\% \\
100 \%\end{array}$ & \multirow{2}{*}{ - } & \\
\hline & Incorrect & 0 & $0.0 \%$ & 0 & $0.0 \%$ & 0 & $0 \%$ & & - \\
\hline \multirow[t]{2}{*}{ Oxygen therapy } & Correct & 37 & $92.5 \%$ & 39 & $97.5 \%$ & 39 & $97.50 \%$ & \multirow{2}{*}{$\begin{array}{c}\text { F. Test } \\
1.053\end{array}$} & \multirow{2}{*}{0.305} \\
\hline & Incorrect & 3 & $7.5 \%$ & 2 & $5 \% 5$ & 2 & $5 \%$ & & \\
\hline \multirow[t]{2}{*}{ Sao2monitoring } & Correct & 40 & $100 \%$ & 40 & $100 \%$ & 40 & $100 \%$ & \multirow[t]{2}{*}{-} & \multirow[t]{2}{*}{-} \\
\hline & Incorrect & 0 & $0.0 \%$ & 0 & $0.0 \%$ & 0 & $0 \%$ & & \\
\hline \multirow[t]{2}{*}{ Arterial puncture } & Correct & 27 & $67.5 \%$ & 40 & $100.0 \%$ & 38 & $95 \%$ & \multirow{2}{*}{$\begin{array}{l}\text { F. Test } \\
15.522\end{array}$} & \multirow{2}{*}{0.000} \\
\hline & Incorrect & 13 & $32.5 \%$ & 0 & $0.0 \%$ & 2 & $5 \%$ & & \\
\hline \multirow[t]{2}{*}{ Cardiac monitoring } & Correct & 36 & $90.0 \%$ & 36 & $90.0 \%$ & 36 & $90 \%$ & \multirow{2}{*}{$\begin{array}{c}\text { F. Test } \\
0.000\end{array}$} & \multirow{2}{*}{1.000} \\
\hline & Incorrect & 4 & $10.0 \%$ & 4 & $10.0 \%$ & 4 & $10 \%$ & & \\
\hline \multirow[t]{2}{*}{ CVP measurement } & Correct & 37 & $92.5 \%$ & 38 & $95.0 \%$ & 38 & $95 \%$ & \multirow{2}{*}{$\begin{array}{c}\text { F. Test } \\
0.213\end{array}$} & \multirow{2}{*}{0.644} \\
\hline & Incorrect & 3 & $7.5 \%$ & 2 & $5.0 \%$ & 2 & $5 \%$ & & \\
\hline
\end{tabular}




\begin{tabular}{|l|l|l|l|l|l|l|l|l|l|}
\hline DVT prophylaxis & Correct & 5 & $12.5 \%$ & 33 & $82.5 \%$ & 31 & $77.50 \%$ & F. Test \\
\cline { 2 - 7 } & Incorrect & 35 & $87.5 \%$ & 7 & $17.5 \%$ & 9 & $22.50 \%$ & 39.298 & 0.000 \\
\hline Neurological management & Correct & 4 & $10.0 \%$ & 36 & $90.0 \%$ & 32 & $80 \%$ & F. Test \\
\cline { 2 - 7 } & Incorrect & 36 & $90.0 \%$ & 4 & $10.0 \%$ & 8 & $20 \%$ & 51.20 \\
\hline
\end{tabular}

NB: ** $\mathrm{P}<.001 \quad$ ns means not significant ${ }^{*} \mathrm{P}<.05 \quad \mathrm{P} 1$ : between pre and post -test $\quad$ FT: Fisher's Exact Test Satisfactory level of nurse's knowledge $=$ score of $75 \%$ and more.

Table 3: reflected comparison between nurses' Knowledge about principals of nursing management of traumatic brain injury on pre, post and follow-up tests. It was revealed that all studied nurses $(100 \%)$ had correct knowledge regarding tracheal suctioning and Sao2 monitoring and majority of them had a satisfactory level of knowledge about Oxygen therapy, Cardiac monitoring and CVP measurement (92.5\%, 90\%, and 92.5\%, respectively). While, there were unsatisfactory level of knowledge about DVT prophylaxis and neurological management $(12.5 \%$ and $10.0 \%$ respectively) on pre-test and improved after providing the educational program.

Table (4):- Nurses' knowledge about basic care of traumatic brain injury on pre, post and follow-up tests. $(\mathbf{n}=\mathbf{4 0})$

\begin{tabular}{|c|c|c|c|c|c|c|c|}
\hline & & \multicolumn{2}{|c|}{ Pre } & \multicolumn{2}{|c|}{ Post } & \multirow{2}{*}{$X^{2}$} & \multirow{2}{*}{ NP value } \\
\hline & & No & $\%$ & No & $\%$ & & \\
\hline \multirow{2}{*}{$\begin{array}{l}\text { Side effect of Increase metabolic } \\
\text { rate }\end{array}$} & Correct & 7 & $17.5 \%$ & 36 & $90.0 \%$ & \multirow{2}{*}{$\begin{array}{l}\text { F. Test } \\
42.288\end{array}$} & \multirow{2}{*}{0.000} \\
\hline & Incorrect & 33 & $82.5 \%$ & 4 & $10.0 \%$ & & \\
\hline \multirow[t]{2}{*}{ Feeding } & Correct & 35 & $87.5 \%$ & 38 & $95.0 \%$ & \multirow{2}{*}{$\begin{array}{l}\text { F. Test } \\
1.409\end{array}$} & \multirow{2}{*}{0.235} \\
\hline & Incorrect & 5 & $12.5 \%$ & 2 & $5.0 \%$ & & \\
\hline \multirow[t]{2}{*}{ Changing position } & Correct & 32 & $80.0 \%$ & 40 & $100.0 \%$ & \multirow{2}{*}{$\begin{array}{l}\text { F. Test } \\
8.889\end{array}$} & \multirow{2}{*}{0.003} \\
\hline & Incorrect & 8 & $20.0 \%$ & 0 & $0.0 \%$ & & \\
\hline \multirow[t]{2}{*}{ Nursing care when ear bleed } & Correct & 5 & $12.5 \%$ & 36 & $90.0 \%$ & \multirow{2}{*}{$\begin{array}{l}\text { F. Test } \\
48.080\end{array}$} & \multirow{2}{*}{0.000} \\
\hline & Incorrect & 35 & $87.5 \%$ & 4 & $10.0 \%$ & & \\
\hline \multirow[t]{2}{*}{ Nursing care to patient eyes } & Correct & 2 & $5.0 \%$ & 34 & $85.0 \%$ & \multirow{2}{*}{$\begin{array}{l}\text { F. Test } \\
51.717\end{array}$} & \multirow{2}{*}{0.000} \\
\hline & Incorrect & 38 & $95.0 \%$ & 6 & $15.0 \%$ & & \\
\hline \multirow[t]{2}{*}{ Contraindication of restrain } & Correct & 40 & $100.0 \%$ & 40 & $100.0 \%$ & \multirow{2}{*}{ - } & \multirow{2}{*}{-} \\
\hline & Incorrect & 0 & $0.0 \%$ & 0 & $0.0 \%$ & & \\
\hline
\end{tabular}

NB: ns means not significant * $\mathrm{P}<.05 \quad \mathrm{P} 1$ : between pre and post- test $\quad$ FT: Fisher's Exact Test

Satisfactory level of nurse's knowledge $=$ score of $75 \%$ and more.

Table 4 illustrated nurses' knowledge about the basic care of traumatic brain injury on pre, post and follow-up tests, it was clarified that on pre-test, all studied nurses (100.0\%) had correct knowledge about contraindication of restraints. Also, the majority of nurses had a satisfactory level of knowledge about feeding and changing position ( $87.5 \%$ and $80.0 \%$ respectively). Although, there were a knowledge deficit about side effect of increase metabolic rate, nursing care when ear bleeds and nursing care to patient eyes $(17.5 \%, 12.5 \%$ and $5.0 \%$,respectively) on pretest. The findings showed that nurse's knowledge improved tremendously on post-test. Therefore, there were highly statistical significant differences between nurses' knowledge at $1 \%$ level of statistical significance. 
Table (5): Nurses' practice regarding Glasgow coma scale on pre, post and follow-up tests. (n=40)

\begin{tabular}{|c|c|c|c|c|c|c|c|c|c|}
\hline \multicolumn{2}{|l|}{ Practice } & \multicolumn{2}{|c|}{ Pre } & \multicolumn{2}{|c|}{ Post } & \multicolumn{2}{|c|}{$\begin{array}{l}\text { Follow } \\
\text { up }\end{array}$} & $\mathbf{X} 2$ & $\begin{array}{l}P \\
\text { value }\end{array}$ \\
\hline \multicolumn{8}{|l|}{ Eye response } & \multirow{3}{*}{$\begin{array}{l}\text { F. Test } \\
5.165\end{array}$} & \multirow{3}{*}{0.023} \\
\hline \multirow[t]{2}{*}{ Observe if the eyes open spontaneously } & Done & 31 & $77.5 \%$ & 38 & $95.0 \%$ & 36 & 90 & & \\
\hline & $\begin{array}{l}\text { Not } \\
\text { done }\end{array}$ & 9 & $22.5 \%$ & 2 & $5.0 \%$ & 4 & 10 & & \\
\hline \multirow[t]{2}{*}{ Test eye opening with painful stimulus } & Done & 11 & $27.5 \%$ & 33 & $82.5 \%$ & 31 & 77.5 & \multirow{2}{*}{$\begin{array}{l}\text { F. Test } \\
24.44\end{array}$} & \multirow{2}{*}{0.00} \\
\hline & $\begin{array}{l}\text { Not } \\
\text { done }\end{array}$ & 29 & $72.5 \%$ & 7 & $17.5 \%$ & 9 & 22.5 & & \\
\hline \multirow[t]{2}{*}{$\begin{array}{c}\text { Assess eye opening, verbally by asking the child to } \\
\text { open his/her eyes }\end{array}$} & Done & 18 & $45.0 \%$ & 37 & $92.5 \%$ & 34 & 85 & \multirow{2}{*}{$\begin{array}{l}\text { F. Test } \\
21.004\end{array}$} & \multirow{2}{*}{22} \\
\hline & $\begin{array}{l}\text { Not } \\
\text { done }\end{array}$ & 22 & $55.0 \%$ & 3 & $7.5 \%$ & 6 & 15 & & \\
\hline \multirow{2}{*}{$\begin{array}{l}\text { Acknowledges if no eye opening even after } \\
\text { application of all previous described stimuli }\end{array}$} & Done & 22 & $55.0 \%$ & 36 & $90.0 \%$ & 34 & 85 & \multirow{2}{*}{$\begin{array}{l}\text { F. Test } \\
12.288\end{array}$} & \multirow[b]{2}{*}{0.000} \\
\hline & $\begin{array}{l}\text { Not } \\
\text { done }\end{array}$ & 18 & $45.0 \%$ & 4 & $10.0 \%$ & 6 & 15 & & \\
\hline \multicolumn{8}{|l|}{ Verbal response } & \multirow{3}{*}{$\begin{array}{l}\text { F. Test } \\
5.165\end{array}$} & \multirow{3}{*}{0.023} \\
\hline \multirow{2}{*}{$\begin{array}{l}\text { Assess child orientation to time, space and aware of } \\
\text { himself }\end{array}$} & Done & 31 & $77.5 \%$ & 38 & $95.0 \%$ & 36 & 90 & & \\
\hline & $\begin{array}{l}\text { Not } \\
\text { done }\end{array}$ & 9 & $22.5 \%$ & 2 & $5.0 \%$ & 4 & 10 & & \\
\hline \multirow{2}{*}{$\begin{array}{l}\text { Test whether the child can answer questions, but } \\
\text { incorrectly, as they are disoriented and confused }\end{array}$} & Done & 35 & $87.5 \%$ & 39 & $97.5 \%$ & 37 & 92.5 & \multirow[b]{2}{*}{$\begin{array}{l}\text { F.Test } \\
2.883\end{array}$} & \multirow[b]{2}{*}{0.090} \\
\hline & $\begin{array}{l}\text { Not } \\
\text { done }\end{array}$ & 5 & $12.5 \%$ & 1 & $2.5 \%$ & 3 & 7.5 & & \\
\hline \multirow[t]{2}{*}{ Identify inappropriate responses from the child's } & Done & 21 & $52.5 \%$ & 36 & $90.0 \%$ & 33 & 82.5 & \multirow[b]{2}{*}{$\begin{array}{l}\text { F. Test } \\
13.730\end{array}$} & \multirow[b]{2}{*}{0.000} \\
\hline & $\begin{array}{l}\text { Not } \\
\text { done }\end{array}$ & 19 & $47.5 \%$ & 4 & $10.0 \%$ & 7 & 17.5 & & \\
\hline Detect incomprehensible speech from the child's & Done & 18 & $45.0 \%$ & 37 & $92.5 \%$ & 32 & 80 & & \\
\hline & $\begin{array}{l}\text { Not } \\
\text { done }\end{array}$ & 22 & $55.0 \%$ & 3 & $7.5 \%$ & 8 & 20 & $\begin{array}{l}\text { F. I lest } \\
21.004\end{array}$ & 0.000 \\
\hline Observe if there is no verbalization of any type & Done & 23 & $57.5 \%$ & 39 & $97.5 \%$ & 37 & 92.5 & & \\
\hline & $\begin{array}{l}\text { Not } \\
\text { done }\end{array}$ & 17 & $42.5 \%$ & 1 & $2.5 \%$ & 3 & 7.5 & $\begin{array}{l}\text { F. Test } \\
18.351\end{array}$ & 0.000 \\
\hline Motor response & & & & & & & & & \\
\hline Observes if the child obeys a command for & Done & 25 & $62.5 \%$ & 36 & $90.0 \%$ & 34 & 85 & F. Test & 0.004 \\
\hline movement. & $\begin{array}{l}\text { Not } \\
\text { done }\end{array}$ & 15 & $37.5 \%$ & 4 & $10.0 \%$ & 6 & 15 & & \\
\hline Observes if there is localization in response to pain & Done & 33 & $82.5 \%$ & 38 & $95.0 \%$ & 36 & 90 & & \\
\hline stimulus & $\begin{array}{l}\text { Not } \\
\text { done }\end{array}$ & 7 & $17.5 \%$ & 2 & $5.0 \%$ & 4 & 10 & $\begin{array}{l}\text { F. Test } \\
3.130\end{array}$ & 0.077 \\
\hline Observe if the child is able to withdraw from pain & Done & 20 & $50.0 \%$ & 36 & $90.0 \%$ & 34 & 85 & Fisher's & \\
\hline & $\begin{array}{l}\text { Not } \\
\text { done }\end{array}$ & 20 & $50.0 \%$ & 4 & $10.0 \%$ & 6 & 15 & $\begin{array}{c}\text { Exact Test } \\
15.238 \mathrm{a}\end{array}$ & 0.000 \\
\hline Observe the abnormal movement (spastic) flexion, & Done & 24 & $60.0 \%$ & 36 & $90.0 \%$ & 33 & 82.5 & & \\
\hline decorticate posture & $\begin{array}{l}\text { Not } \\
\text { done }\end{array}$ & 16 & $40.0 \%$ & 4 & $10.0 \%$ & 7 & 17.5 & $\begin{array}{l}\text { F. Test } \\
9.600\end{array}$ & 0.002 \\
\hline Detect extensor (rigid) response $\&$ decerebrate & Done & 25 & $62.5 \%$ & 37 & $92.5 \%$ & 34 & 85 & & \\
\hline posture & $\begin{array}{l}\text { Not } \\
\text { done }\end{array}$ & 15 & $37.5 \%$ & 3 & $7.5 \%$ & 6 & 15 & $\begin{array}{l}\text { F. Test } \\
10.323\end{array}$ & 0.001 \\
\hline $\begin{array}{c}\text { Identify if no response even after application of all } \\
\text { previous described stimuli. }\end{array}$ & Done & 25 & $62.5 \%$ & 36 & $90.0 \%$ & 33 & 82.5 & F. Test & $0 \cap 004$ \\
\hline & $\begin{array}{c}\text { Not } \\
\text { done }\end{array}$ & 15 & $37.5 \%$ & 4 & $10.0 \%$ & 7 & 17.5 & 8.352 & 0.004 \\
\hline
\end{tabular}

NB: ** $\mathrm{P}<.001 \quad$ ns means not significant ${ }^{*} \mathrm{P}<.05 \quad \mathrm{P} 1$ : between pre and posttest $\quad$ FT: Fisher's Exact Test Satisfactory level of nurse's knowledge $=$ score of $75 \%$ and more.

Table 5 represented comparison between nurses' practice regarding GCS on pre, post and follow-up tests, as showed in the table, the majority of studied nurses had incompetent practice $<85 \%$ on pre-test. However, on posttest, all nurses had competent practice $<85 \%$. Therefore, there were highly statistical significant differences between practices of nurses at $1 \%$ level of statistical significance. 
Table (6) Mean of nurses' practice about Glasgow coma scale on pre and post-tests

\begin{tabular}{|l|l|l|l|l|}
\hline Practice & Pre & Post & Follow up & \multirow{2}{*}{ F.test } \\
\cline { 3 - 4 } & & Mean \pm S.D & $\begin{array}{l}\text { Mean } \pm \\
\text { S.D }\end{array}$ & \\
\cline { 4 - 5 } & & & & \\
\hline Eye response & $2.35 \pm 1.25$ & $3.58 \pm 0.68$ & $3.22 \pm 0.76$ & 143.25 \\
\hline Verbal response & $3.20 \pm 1.54$ & $4.73 \pm 0.51$ & $4.23 \pm 0.49$ & 141.28 \\
\hline Motor response & $3.80 \pm 1.94$ & $5.48 \pm 0.91$ & $5.21 \pm 0.86$ & 144.9 \\
\hline
\end{tabular}

Table 6 showed Mean of nurses' practice about Glasgow coma scale on pre, post-tests and follow up tests. It was revealed that there were prominent improvements of Mean \pm S.D in the scores of practices about Glasgow coma scale on post and follow-up test. Therefore, there were highly statistical significant differences between nurses' knowledge and practice at $1 \%$ level of statistical significance.

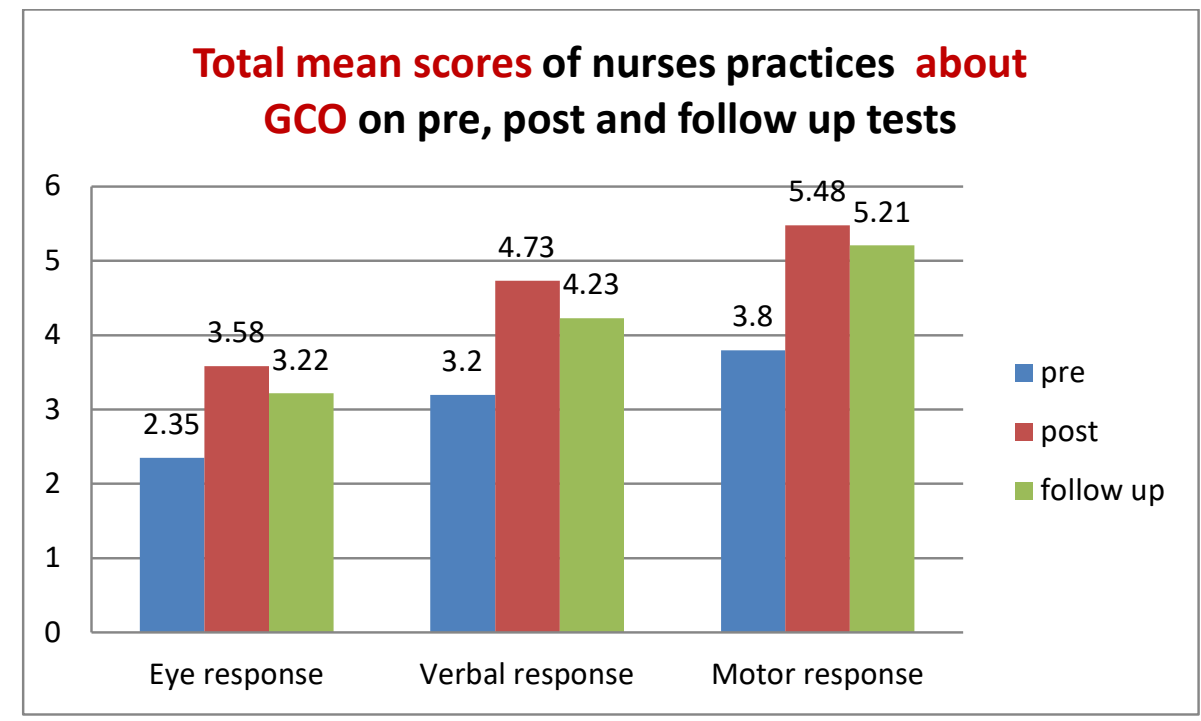

Figure (5): Total mean scores of nurses' practices about Glasgow coma scale on pre, post-tests and follow up tests.

Figure 5 : showed total mean scores of nurses' practices about Glasgow coma scale on pre, post and follow up tests, it was represented that there was a reduction in total practice score after three months in all items than on post-test.

\section{Discussion}

The results of the present study support the hypotheses that nurses' knowledge was improved after educational nursing strategies on post and follow up tests rather than on pre-test. Also, nurses' skills were improved and there are positive association between nurses' Knowledge and skills post implementation of educational nursing strategies. Concerning demographical characteristic of studied sample, it was illustrated that mean \pm SD were 29.1 \pm 6.36 . This was supported by Mahday et al., (2016) who reported that the mean age of the nurses under the study was $32.7 \pm 4.5$. This was contradicted with Oyesanya, (2018) who mentioned that mean age of studied nurses was 40.3 years. This could show that the majority of nurses had limited experience especially in management of children with traumatic brain injury. So, enhancing nurse's level of knowledge through in-service education was promptly needed. Also, the study showed that more than half of studied nurses (55\%) aged between $20-<30$ years old and $42.5 \%$ of them were aged $30<40$ years old. This was similar to Abd El Moteleb (2014) who reported that more than two third of nurses age less than thirty years. Also, Shehab et al., (2018) mentioned that more than two third of them had 20-30 years old. This may illustrate needs for in-services training sessions to enhance nurses knowledge and performance to promote patient safety and improve quality of life. 
Regarding educational level, it was clarified that the majority of studied nurses had techniqal nursing institute and bachelor degree ( $43 \%$ and $40 \%$ respectively) while, the minority of them were diploma nursing (17\%). Such finding was in agreement with Hussein, (2018) who reported that $51.1 \%$ of them had a technical nursing education. In this respect, Eldesouky, (2016) reported that $63.9 \%$ of studied nurses were graduated from secondary nursing school and more than one third of them (36.1\%) graduated from technical nursing institute. This was contradicted with Oyesanya et al., (2018) who mentioned that nursing diploma were $2.0 \%$ and bachelors' degree were $67.8 \%$, while masters or doctorate degree were 16.1\%. Also, this was reversed with Seliman et al., (2014) who reported that $6 \%$ of the study subjects were technical nursing institute, while the majority $(94 \%)$ of them have diploma degree. This could show that enhancing nurse's level of education through in-service education was promptly needed.

Concerning nurses' years of experience, it was showed that $37.50 \%$ of studied nurses had $5-<10$ years and $25 \%$ of them had either $1-<5$ or $15-<20$ years of experience. In this respect, Hussein (2018) mentioned that $66.7 \%$ of studied nurses had $5-<10$ years of experience. This demonstrates needs for improvement of knowledge and performance regarding traumatic brain injury. According to previous training in traumatic brain injury, it was clarified that none of nurses had previous training in traumatic brain injury. This was consistent with Shehab et al., (2018) who reported that all of studied nurses had no training and also there is no protocol of care. In this respect, Refai (2012) indicated that most of studied nurses had no training. This result was congruent with Oyesanya (2019) who reported that only 7\% of nurses received training as part of their education. In this regards, Hussein (2018) emphasized that only $28.9 \%$ of them had previous training regarding management of head injury. This was consistent with Choudhary (2009) who mentioned that appropriate preparation of nurses is a vital component in providing quality care to child's and their families.

The findings represented differences in Mean \pm S.D of nurses' knowledge about traumatic brain injury on pre, post and follow up tests. It was clarified that nurses of our study showed that total Mean \pm S.D of knowledge increased on post and follow up tests rather than on pre-test. This was in line with Hussein (2018) who reported that there was improvement in the total score of knowledge post educational program. This was supported with Ghoneim et al., (2012) who mentioned that implementation of nursing care protocol on moderate head injured patient had best effect on minimizing the incidence of all systemic complications, decrease morbidity and mortality rate. The most prominent improvements of Mean \pm S.D were in the scores of knowledge about Glasgow coma scale and principals of nursing care $(8.30 \pm 1.11$ and $8.30 \pm 1.11$ respectively). This was in line with Reith et al., (2016) who reported that majority of nurses showed good knowledge about GCS. Therefore, there were highly statistical significant differences between nurses' knowledge on pre, post and follow up tests at $1 \%$ level of statistical significance. This was supported by with Shahin et al., (2012) who stated that a highly statistical significant correlation between participants' scores of knowledge in pre-program and post program following the instructional program. This could be attributed to the implementation of educational program which helped to increase nurse's awareness of traumatic brain injury.

On the other hand, findings of the current study reported a gradual decrement in nurses' knowledge by time after three months post program implementation. This was in line with Mansour (2014) who emphasized that the result reporting a decline with limited value in nurse's knowledge level after 3 months period, than immediately after the program implementation. Therefore, nurses needed a continuous training program in TBI to improve their knowledge and performance. This was supported by Shehab et al., (2018) who mentioned that developing system of periodical nurse's evaluation is very important to update their knowledge and enhancing their practice. Our findings also represented comparison between nurses' Knowledge about principals of nursing management of traumatic brain injury on pre, post and follow-up tests. It was revealed that all studied nurses had correct knowledge regarding tracheal suctioning and Sao2 monitoring (100\%) and majority of them had satisfactory level of knowledge about oxygen therapy, cardiac monitoring and CVP measurement $(92.5 \%$, 90\%, and $92.5 \%$ respectively). This indicates that the higher level of education is associated with higher knowledge score and higher competence in the application of procedures.

The present study revealed that there were unsatisfactory level of knowledge about DVT prophylaxis and neurological management ( $12.5 \%$ and $10.0 \%$ respectively). This demonstrates need for training session to enhance nurse's knowledge and to clarify the role of the nurses in caring for children with TBI. This was corresponding with Oyesanya et al., (2016) who mentioned that more education and training is needed for nurses to ensure they have factual information about TBI and to clarify their roles. This was consistent with the findings done by McQuillan \& Mitchell (2002) who stated that specialized training and education about caring for children with TBI provides the nurse with knowledge to interpret the child's assessments and corresponding treatments, current evidence-based practice guidelines and strategies for collaborating with the interdisciplinary team. 
Also, the findings showed that nurse's knowledge had improved tremendously after providing educational program. Therefore, there were highly statistical significant differences between nurses' knowledge at $1 \%$ level of statistical significance. This was corresponding with Hussein (2018) who indicated that there were high statistical significant differences in knowledge scores throughout the program intervention among studied nurses $(p<0.001)$. Hence research hypothesis stated that the mean post-test knowledge scores of the staff nurses will be significantly higher than the mean pre-test knowledge scores. The improvement of nurses' knowledge results from implementation of educational program. This result was consistent with the findings of Abdelmowla (2015) who reported that enhancement of nurses' knowledge is a consequence of implementing a training program. In this respect, Seliman et al., (2014) mentioned that there are an obvious improvement in nurses knowledge scores were documented post protocol implementation as compared to their pre protocol with highly significant statistically differences. This could reflect that nurses were in need for a serious of continuing training program and followups to adapt to new knowledge and practices.

Concerning nurses' knowledge about basic care of traumatic brain injury on pre, post and follow-up tests, it was clarified that on pre-test, all studied nurses had correct knowledge about contraindication of restraints $(100.0 \%)$. Also, the majority of nurses had satisfactory level of knowledge about feeding and changing position ( $87.5 \%$ and $80.0 \%$ respectively). This may be associated with more work experience had effect on their knowledge. Although, there were unsatisfactory level of knowledge about side effect of increase metabolic rate, nursing care when ear bleed and nursing care to pt. eyes (17.5\% and $12.5 \%$ and $5.0 \%$ respectively). Perhaps this can be attributed to lack of in-service education. Therefore, there is a need for continuous educational programs to update nurse's knowledge. This was corresponding with Tume (2007) who mentioned that it is important for nurses working with children with traumatic brain injuries to understand the neuropathophysiology of pediatric traumatic brain injury and the evidence for nursing interventions used in the intensive care.

Also, the findings showed that nurse's knowledge improved tremendously after providing them educational programs about traumatic brain injury. This result is consistent with Eldesouky, (2016) who confirmed that improved nurse's information leads to improved knowledge and performance which contribute to the quality of care. Therefore, there were highly statistical significant differences between nurses' knowledge at $1 \%$ level of statistical significance. This was in line with Shehab et al., (2018) who reported that there were high statistical significant differences in knowledge scores of traumatic brain injury throughout the program intervention. In addition, our finding represented comparison between nurses' practice regarding GCS on pre, post and follow-up tests, it was represented that the majority of studied nurses had incompetent practice $<85 \%$ on pre-test. This was consistent with Thi and Chae (2011) who concluded that the nurses were lacking practices about GCS especially when it comes to the clinical setting. As well as Fischer and Mathieson (2001) reported an inconsistency in the use of GCS in the clinical setting. This may be due to shortage of training programs therefore education and training is required to ensure that the tool is used as a valid indicator of patient status.

However, on post-test, all nurses had competent practice $<85 \%$ of total practice score. Therefore, there were highly statistical significant differences between practices of nurses at $1 \%$ level of statistical significance. This is supported by the findings of other similar study conducted with Eldesouky (2016) who reported that there are statistically significant improvements immediately after program implementation of GCS. This is comparable with Shahin et al., (2012) and Mohammed \& Taha (2014) who stated that there were a highly statistical significant correlation between participants' scores of practice in pre-program and post program following the instructional program. This was in line with Hussein (2018) who concluded that educational intervention had a positive effect in improving nurses' practice regarding head injury in intensive care unit. There was also a positive correlation between levels of nurses' knowledge as regards their practice.

Moreover, it was also found that statistically significant relation between nurses' knowledge and practice. Thereby, improvements of specific knowledge related to traumatic brain injury are associated with achievement of best practice and increase precision in the use of scale. Therefore, there were highly statistical significant differences between practices of nurses at $1 \%$ level of statistical significance. This agree with Mohammed \&Taha (2004) and Seliman (2014) who stated that a highly statistical significant correlation between participants' scores of practice in pre-program, post program, 1 month and 2 months following the instructional program. In the present study, the most prominent improvements of Mean practice score about motor response $(5.48 \pm 0.91)$. Therefore, there were highly statistical significant differences between nurses' practice at $1 \%$ level of statistical significance. On the other hand, Hussein (2018) concluded that majority of studied subjects had satisfactory level of knowledge at both immediate post interventions and follow up phases $(91.1 \%$ and $84.4 \%$ respectively). 
This was agreed with Abd el-Aziz (2014) who concluded that the education program leads to significant improvement in nurse's skills. This finding was congruent with Taha (2004) who reports an improvement after implementation of the program with a highly significant statistical difference. These agree with Shahin et al., (2012) who stated that a highly statistical significant correlation between participants' scores of knowledge in pre-program and post program following the instructional program. This improvement revered to quality of the educational nursing strategies. On follow-up tests, it was noticed from the results of the present study that there was a reduction in total practice scores obtained after three months in some items than on immediate post-test. In this respect, Mansour (2014) emphasized that the result of his study reported a decline with limited value after 2 months period, than immediately after the program implementation. This could reflect that nurses need for a series of continuing training program and adequate follow-ups to adapt to new knowledge and practices.

\section{Conclusion}

In the light of the present study, it was concluded that knowledge and skills of nurses had significantly improved after providing teaching program as evident from the obtained data $\mathrm{P}<0.001$.

\section{Recommendations}

Based on the results of this study the following recommendations are suggested:-

○ The study recommended constant educational programs on a regular basis for nurses who care for traumatic brain injury for enhancing high quality of life.

o Updating knowledge and practice of newly joined nurses about evidence-based care bundle for traumatic brain injury to improve their knowledge and practice.

- Ensure that educational hand-out more accessible to hospitals for nurses in order to remind themselves on assessment, treatment, prevention of complications and variety of care provided.

o Prevention is a positive attitude towards reduction of traumatic brain injuries in children through public education and implementation of safety approaches.

o Similar study can be replicated on a large sample to generalize the findings.

\section{Acknowledgment}

We are sincerely thankful for nursing staff in the Pediatric Intensive Care Units and Emergency Department at Birket El Sabah Central Hospital for participating and to complete the study despite their overwhelming duties. Finally, we are grateful to everyone helped us to complete this work.

\section{References}

○ Abd El- Moteleb, H. (2014). Effect of Training Program on Nurses' Performance for Caring of Patients with Traumatic Brain Injury. Doctorate Thesis in Medical Surgical Nursing Faculty of Nursing. Ain Shams University. Retrieved from https:// www. ncbi. nlm. nih. gov/pmc /articles/PMC3088371

- Abd El-Aziz, M. (2014). Effect of educational program on nurses' knowledge and skills about oral care for traumatized patients. AL-AzharAssiut Medical Journal AAMJ, 12(1), 25-45.

○ Abdelmowla, R., El-Lateef, Z and El-Khayat, R. (2015). Impact of nursing educational program on reducing or preventing Postoperative complication for patient after Intracranial Surgery. Journal of Educational and Practice, 6(9).

- Alsolamy, S., Alotaibi, F., Arabi, Y.(2017). Knowledge gaps in the global practice of management of severe traumatic brain injury. King Saud Bin Abdulaziz University for Health Sciences, King Abdulaziz Medical City.

- Amaranath, M., Ramanan, J., Reagh, E., Saekang, N., Prasad, R and Chaseling, S. (2014). Epidemiology of traumatic head injury from a major paediatric trauma centre in New South Wales, Australia ANZ Journal of Surgery, pp. 424-428, 10.1111/ans.12445 CrossRefView Record in Scopus, Google Scholar

- Benson BW, Meeuwisse WH, Rizos J, Kang J, Burke CJ.(2011). A prospective study of concussions among National Hockey League players during regular season games: the NHL-NHLPA concussion program. CMAJ. 2011;183(8):905-11. Google Scholar

○ Borich, M., Cheung, K., Jones, P., Khramova, V., Gavrailoff, L and Boyd, L. (2013). Concussion: current concepts in diagnosis and management. J Neurol Phys Ther. 2013; 37 (3):133-9. Google Scholar 
- Choudhary, KA. (2009). Study to assess the effectiveness of Structured teaching program on knowledge regarding care of head injury patients among staff nurses in a selected Hospital at Bangalore. Thesis. Rajiv Gandhi University of Health Sciences, Adarsha College of Nursing.

- Das, A., Botticello, AL., Wylie, GR and Radhakrishnan, K. (2012). Neurologic disability: A hidden epidemic for India. Neurology. [PMC free article] [PubMed] [Google Scholar]

○ $\quad$ Dwyer, B and Katz, DI. (2018). Postconcussion syndrome. Handb Clin Neurol. 2018;158:163-78.PubMed

- Egyptian Central Agency for Mobilization and Statistic. (2016). Available at: http: //www.capmas.gov.eg.

- Elhagga, I and Eldesouky, E. (2016). Impact of an Educational Program for Nurses' Knowledge and Practice about Glasgow Coma Scale. Medical Surgical Nursing Department, Faculty of Nursing, Port Said University, Republic Arabia Egypt. International Journal of Recent Scientific Research Vol. 7, Issue, 3, pp. 9690-9695.

- El-Naggar, M. (2014). Pediatric critical care, university Tumkur. $1^{\text {ST }}$ ed. Nursing Medical Surgical book center, Faculty of Medicine, Cairo University, Nursing, Aruna College of Nursing, Tumkur. Cairo Egypt

○ Falk A, Alm, A \& Lindstrom V. (2014): Has increased nursing competence in the ambulance services impacted on pre-hospital assessment and interventions in sever traumatic brain-injured patients? Journal of Trauma, Resuscitation and Emergency Medicine 22:20.

- Faul, M., Xu, L., Wald, M., Coronado, V. (2010). Traumatic brain injury in the United States: emergency department visits, hospitalizations and deaths 2002-2006. Centers for Disease Control and Prevention, National Center for Injury Prevention and Control; Atlanta, GA: 2010

○ $\quad$ Finnie, JW and Blumbergs, PC. (2002). Traumatic brain injury. Vet Pathol 2002; 39:679-89. In Maas, AI., Murray, GD., Roozenbeek, B., Lingsma, HF., Butcher, I and McHugh GS, et al. (2013). Advancing care for traumatic brain injury: Findings from the IMPACT studies and perspectives on future research. Lancet Neurol 2013;12:1200-10

- Fischer, J and Mathieson, C. (2001).The history of the Glasgow Coma Scale: implications for practice. Crit Care Nurs Q 23: 52-58.In Falk, A. (2015). Educational Intervention to Improve Nursing Practice in the Critical Care Setting. International Archives of Nursing and Health Care

○ Garg, K., Sharma, R., Gupta, D., Sinha, S., S Satyarthee, G., Agarwal, D., ～Kale, S and Mahapatra, A. (2017). Outcome Predictors in Pediatric Head Trauma: A Study of Clinicoradiological Factors. J Pediatr Neurosci. 2017 Apr-Jun; 12(2): 149-153. doi: 10.4103/jpn.JPN_179_16. Retrieved from https:// www. ncbi. nlm. nih. gov/ pmc/articles/PMC5588639/

○ Gennai, A., Monsel, Q., Hao, J., Liu, V., Gudapati, E., Barbier, J. W and Lee, S. (2015).Cell-Based therapy for traumatic brain injury. British Journal of Anaesthesia115 (2): 203-12

○ Ghoneim, N., Alaa Elden, S., Okab, M., and Elsaay, O. (2012). Impact of implementing nursing care protocol on moderate head injured patient's outcome. Journal of American Science; 8(7):649- 664. http://www.jofamericanscience.org

○ Godwin, J.S. Kreutzer, J.C. Arango-Lasprilla, T and Lehan, J. (2011).Marriage after brain injury: Review, analysis, and research recommendations The Journal of Head Trauma Rehabilitation, 26 (2) (2011), pp. 4355 View Record in ScopusGoogle Scholar

○ Granacher, RP. (2015). Traumatic Brain Injury: Methods for Clinical and Forensic Neuropsychiatric Assessment. $3^{\text {rd }}$ ed. Riyadh, Saudi Arabia: CRC Press; 2015.

- Haddad, S and Arabi, Y. (2012). Critical care management of severe traumatic brain injury in adults. Emergency Medicine. Retrieved from http: //www.s jtrem. com/content/20/1/12

○ Hassanein, M., Adam, F., Elbalam, K and Abubeih, H. (2018). Epidemiology of Hospital stay, morbidity and mortality in polytrauma patients in Assiut University HospitaL. Assiut University. Faculty of Medicine. Retrieved from .eulc.edu.eg/eulc.

○ Hemphill, JC and Phan, N.(2016). Management of acute severe traumatic brain injury. Uptodate. 2016:127. [Google Scholar]

○ Heugten, C., Renaud, I \& Resch, C. (2017). The role of early intervention in improving the level of activities and participation in youths after mild traumatic brain injury: a scoping review. Future science group. 10 Aug 2017https://doi.org/10.2217/cnc-2016-0030 
○ Hopkins, S., Vachhrajani, J., Tymianski, D., Petrelli, T and White, E. (2016). Guidelines for Basic Paediatric Neurological Observation. Neurosurgery Education and Outreach Network (NEON) Version 1.0 | Critical Care Services Ontario. Retrieved from https://www.criticalcareontario.ca

- Hussein, E. (2018). Intensive Care Unit Nurses' Performance Regarding Caring Patients with Head Injury: An Educational Intervention. Medical Surgical Nursing, Faculty of Nursing, Zagazig University, Egypt. International Journal of Studies in Nursing; Vol. 3, No. 3; 2018

- Ismail, A., Shenouda, A and Tharwat, A. (2019). Levetiracetam versus phenytoin for seizure prophylaxis in patient with traumatic brain injury. Ain Shams University. Faculty of Medicine. Intensive Care department. Retrieved from .eulc.edu.eg/eulc.

- Jaddoua, B., Mohammed, W., and Abbas, A. (2013). Assessment of Nurse's Knowledge Concerning Glasgow Coma Scale in Neuro Surgical Wards. Journal of Kufa for Nursing Science. 3 (2) 1-10. In AlQuraan, H \& AbuRuz, M. (2016). Assessment Of Jordanian Nurses' Knowledge To Perform Glasgow Coma Scale. European Scientific Journal September 2016 edition vol.12, No.27 ISSN: 1857 - 7881.

- Koepsell, TD., Rivara, FP and Vavilala, M. (2017). Incidence and descriptive epidemiologic features of traumatic brain injury in King County, Washington. Pediatrics 128(5), 946-954 (2011).Google Scholar

- Kutzleb, J. (2012). Evidence-Based Practice Nursing Interventions for Improved Functional and Cognitive Outcomes in the Traumatic Brain Injury Patient. Retrieved from http://dx.doi.org/10.4172/21671168.1000110

○ Langlois JA., Rutland-Brown, W and Thomas KE. (2004). Traumatic Brain Injury in the United States: Emergency Department Visits, Hospitalizations, and Deaths, Centers for Disease Control and Prevention, National Center for Injury Prevention and Control, Atlanta 2004. In Schutzman, S. (2018). Minor head trauma in infants and children: Evaluation. Jun 2019. | This topic last updated: Dec 18, 2018.

○ Levin, HS., Hanten, G and Roberson, G. (2008). Prediction of cognitive sequelae based on abnormal computed tomography findings in children following mild traumatic brain injury. J. Neurosurg. Pediatr. 1(6), 461-470 (2008).

○ Maas, AI., Murray, GD., Roozenbeek, B., Lingsma, HF., Butcher, I and McHugh, GS. (2013). Advancing care for traumatic brain injury: Findings from the IMPACT studies and perspectives on future research. Lancet Neurol 2013;12:1200-10

- Maconochie I. (2003). Accident Prevention. BMJ. 2003; 88 (4):275-7. 2. In Elbai, A., Saker, T., Aziz, $\mathrm{S}$ and Shahate, A. (2016). Patterns of extremities trauma in children and their management in emergency department in Suez Canal University Hospital, Ismailia, Egypt. International Surgery Journal. DOI: http://dx.doi.org/10.18203/2349-2902.isj20161163.

- Mahday, N., Mersal, N and Hessien, Z. (2016). Increased Intracranial Pressure: Effect of Educational Nursing Guidelines on Nurses' Performance in Neuro-Critical Care Unit. Life Science Journal. Retrieved from http://www.lifesciencesite.com

○ Mansour, H. (2014). The effect of implementing triage training competencies on newly graduated nurses (NGNs) working in emergency department at emergency hospital. University of Mansoura, Faculty of Nursing, Egypt

- McCrory P, Meeuwisse W, Dvorak J, Aubry M, Bailes J, Broglio S. (2017). Consensus statement on concussion in sport-the 5(th) international conference on concussion in sport held in Berlin, October 2016. Br J Sports Med. 2017;51(11):838-47.

- McNarry AF, Goldhill DR. Simple bedside assessment of level of consciousness: Comparison of two simple assessment scales with the Glasgow coma scale. Anaesthesia. 2004;59(1):34-37

- McQuillan KA and Mitchell PH. (2002). Traumatic brain injuries. In: McQuillan KA, Von Rueden K, Hartstock RL, Flynn MB, Whalen E, editors. 3rd edition W. B. Saunders Company; Philadelphia, PA: 2002. pp. 394-461. [Google Scholar]. In Oyesanya,T., Brown, R and Turkstra, L. (2017). Caring for Patients with traumatic brain injury: a survey of nurses' perceptions.Journal of nursing and management. 27 June 2016. https: //doi. org/ 10. 1111 /jocn.13457

○ Mohammed, EK and Taha, AS. (2014): Critical care nurses' knowledge and practice regarding administration of total parenteral nutrition at critical care areas in Egypt. Journal of Biology, Agriculture and Healthcare; 4(13): 10-22. 
- Morin M, Langevin P, Fait P. Cervical Spine Involvement in Mild Traumatic Brain Injury: A Review. J Sports Med (Hindawi Publ Corp). 2016;2016:1590161. Google Scholar

- Mucha A, Collins MW, Elbin RJ, Furman JM, Troutman-Enseki C, DeWolf RM, et al. A brief vestibular/ocular motor screening (VOMS) assessment to evaluate concussions: preliminary findings. Am J Sports Med. 2014;42(10):2479-86.PubMed

○ Murthy T, Bhatia P, Sandhu K, Prabhakar T, Gogna R. (2005). Secondary brain injury: Prevention and intensive care management. Indian J Neurotrauma. 2005; 2:7-12. [Google Scholar]

- National institute for health and clinical excellence. (2013). Head injury Triage, assessment, investigation and early management of head injury in infants, children and adults.

○ Neumann, V., Gutenbrunner, Ch., Fialka-Moser, V., Christodoulou, N., Varela, E., Giustini, A and Delarque, A. (2010). Interdisciplinary Team Working in Physical and Rehabilitation Medicine. Journal of Rehabilitation Medicine, Volume 42, Number 1, January 2010;42(1): 4-

8 DOI: https://doi.org/10.2340/16501977-0483

○ Oyesanya TO, Thomas MA, Brown RL, Turkstra, LS. (2016). Nurses' Beliefs About Caring for Patients With Traumatic Brain Injury. West J Nurs Res. 2016 Sep;38(9):1114-38. doi: 10.1177/0193945916636629. Epub 2016 Mar 7. PMID: 26955844

○ Oyesanya, T., Brown, R and Turkstra, L .(2017). Caring for Patients with traumatic brain injury: a survey of nurses' perceptions.Journal of nursing and management. 27 June 2016. https: //doi. org/ 10. 1111 /jocn.13457

- Oyesanya, T and Snedden, T. (2018). Pediatric Nurses' Perceived Knowledge and Beliefs of EvidenceBased Practice in the Care of Children and Adolescents with Moderate-to-Severe Traumatic Brain Injury. J Spec Pediatr Nurs. 2018 April ; 23(2): e12209. doi:10.1111/jspn.12209.

○ Oyesanya, T. (2019). Veterans Health Administration nurses' training and beliefs related to care of patients with traumatic brain injury. Retrieved from https: //doi. org/ 10. 1371 /journal.pone.0222585

- Paul, M. (2012). Practical use of the Glasgow Coma Scale; a comprehensive narrative review of GCS methodology. Australasian Emergency Care Journal. August 2012.Volume 15, Issue 3, Pages 170-183

○ Ponsford J, Nguyen S, Downing M, Bosch M, McKenzie JE and Turner S. (2019). Factors associated with persistent post-concussion symptoms following mild traumatic brain injury in adults. J Rehabil Med. 2019; 51(1):32-9. PubMed

- Prado, C. (2007). Teaching-learning of the Glasgow Coma Scale: analysis of two techniques among nurses in emergency department. . Sao Carlos Informatics Center. [Google Scholar]

○ Refai, A. (2012): Impact of a designed teaching protocol about advanced cardiac life support (ACLS) on critical care nurse's knowledge and practices at Benha University Hospital, Cairo, Egypt. Journal of American Science; 8(10):838-850.

- Reith, FC., Brennan, PM., Maas, A and Teasdale, GM. (2016). Lack of Standardization in the Use of the Glasgow Coma Scale: results of International Surveys. J Neurotrauma. 2016;33(1):89-94.

- Satyanegara. (2010). Potential utilization of nursing teleconsultation in improving traumatic brain injury patient's quality of life in West Java, Indonesia. Online Journal of Nursing Informatics (OJNI), 22(2), Available at http://www.himss.org/ojni

- Seliman, A. (2015). Impact of a designed head trauma nursing management protocol on critical care nurses' knowledge and practices at emergency hospital Mansoura university.

- Seliman, M.A., Morsy, Y.W., Sultan, A.M., Elshamy, K.F.S., \& Ahmed, H. (2014). Impact of a Designed Head Trauma Nursing Management Protocol on Critical Care Nurses' Knowledge and Practices at Emergency Hospital Mansoura University. Journal of American Science, 10(12s). Retrieved from http:// www. Jof american science. org

○ Shahin, M.A., Mohamed, W.Y., \& Sayed, M. (2012). Nurses' knowledge and practices regarding enteral nutrition at the critical care Department of Al-Manial University Hospital in Egypt: impact of a designed instructional program. J. Am. Sci, 8(11), 397-405.

○ Shehab, S.M., Ibrahim, M.N., \& Abd-Elkader, H. (2018). Impact of an Educational Program on Nurses' Knowledge and Practice Regarding Care of Traumatic Brain İnjury Patients at Intensive Care Unit at Suez Canal University Hospital. International Journal of Caring Sciences, 11(2), 1104. 
- Sheriff, FG and Hinson, HE. (2015). Pathophysiology and clinical management of moderate and severe traumatic brain injury in the ICU. Semin Neurol. 2015;35:42-9. [PMC free article] [PubMed] [Google Scholar]

- Taha, N. (2004): Comatosed patients: impact of a training program provided for nurses working in critical care units, Zagazig University Hospitals on nurses' knowledge and performance levels as well on patient's outcome.University of Zagazig, Faculty of Nursing, Egypt.

○ Teasdale, G., Maas, A., Lecky, F., Manley, G., Stocchetti, N., \& Murray, G. (2014). The Glasgow Coma Scale at 40 years: Standing the test of time. The Lancet Neurology, 13(8), 844-854. https://doi.org/10.1016/ S1474-4422(14)70120-6

- Thi, N and Chae, H. (2011). The Accuracy of Glasgow Coma Scale Knowledge and Performance among Vietnamese Nurses. Perspectives In Nursing Science,8, 54-61.

$\circ \quad$ Tume, L. (2007). The Nursing management children with severe traumatic brain injury and raised ICP in intensive care. Journal of Neuroscience Nursing. Retrieved from https: //www. Researchgate.net/publication/230692489

- Unterberg AW, Stover JF, Kress B, Kiening KL: Edema and brain trauma. Neuroscience. 2004, 129: 10219. Google Scholar. In Haddad, S and Arabi, Y. (2012). Critical care management of severe traumatic brain injury in adults. Emergency Medicine 2012,. Retrieved from http: //www.s jtrem. com/content/20/1/12

○ Van Pelt ED, De Kloet A, Hilberink SR et al. (2011). The incidence of traumatic brain injury in young people in the catchment area of the University Hospital Rotterdam, The Netherlands. Eur. J. Paediatr. Neurol. 15(6), 519-526 (2011).Google Scholar

○ Widerstrom, E., Finnerup,N and Siddall, P.(2009). Biopsychosocial perspective on a mechanisms-based approach to assessment and treatment of pain following spinal cord injury. Journal of rehabilitation research \& development. Volume 46 Number 1, 2009

- Yattoo GH, Tabish SA, Afzal WM, Kirmani A. (2009). Factors influencing outcome of head injury patients at a tertiary care teaching hospital in India. Int J Health Sci (Qassim) 2009;3:59-62.in Alsolamy, S., Alotaibi ,F., Arabi, Y.(2017). Knowledge gaps in the global practice of management of severe traumatic brain injury. King Saud Bin Abdulaziz University for Health Sciences, King Abdulaziz Medical City.

○ Zemek, RL., Farion, KJ., Sampson M., McGahern, C. (2013). Prognosticators of persistent symptoms following pediatric concussion: a systematic review. JAMA Pediatrics. 2013; 167(3):259-265. [PubMed: 23303474]. 